\title{
Pneumococcal pneumonia-a history based on chapters from the first edition (1950) and the latest edition (2018) of Harrison's Principles of Internal Medicine
}

\author{
Jonathan J Kopel BS, Ali Hakim MD, Kenneth Nugent MD, Steven L. Berk MD
}

\begin{abstract}
Streptococcus pneumoniae remains an important cause of pneumonia-related morbidity and mortality, especially in children and older patients. Although routinely encountered in clinical practice, many clinicians do not know the historical developments in the management of pneumococcal pneumonia and their impact on public health interventions. Reviewing the evolution of clinical information about pneumococcal pneumonia can provide insight into the scientific and technological advances in medicine. Specifically, the development of antibiotics and the development of vaccines against pneumococci were important advances in modern infectious disease control. In addition, the emergence of antibiotic resistant bacteria marks an important development and highlights the need for new antibiotics, antibiotic stewardship, and effective vaccination. To illustrate these ideas, we discuss the evolution of clinical information about pneumococcal pneumonia between the $1^{\text {st }}$ edition (published in 1950) and $20^{\text {th }}$ edition (published in 2018) of Harrison's Principles of Internal Medicine. This comparison outlines the important advances in the prevention and treatment of pneumonia and provides the background for understanding future challenges in identifying, treating, and preventing bacterial infections, such as pneumococcal pneumonia.
\end{abstract}

Keywords: Pneumococcal pneumonia, Harrison's Principles of Internal Medicine, antibiotics, vaccination, serotypes

\section{INTRODUCTION}

Louis Pasteur and George Sternberg first identified Streptococcus pneumoniae by injecting human saliva into rabbits to create experimental infections in the late $19^{\text {th }}$ century. ${ }^{1,2}$ This bacterium was subsequently identified as an important cause of pneumonia, and it potentially caused approximately one half of the pneumonia deaths in the United States in the first third of the $20^{\text {th }}$ century. Pneumococcal pneumonia has been studied extensively by microbiologists and clinicians. For example, early work included

Corresponding author: Jonathan Kopel

Contact Information: Jonathan.Kopel@ttuhsc.edu

DOI: $10.12746 /$ swrccc.v9i37.803
Benjamin White's 1938 book entitled The Biology of the Pneumococcus with 1,593 references, and Roderick Heffron's book Pneumonia, with Special Reference to Pneumococcus Lobar Pneumonia with 1,472 references; both provided detailed information about the bacteriological and clinical aspects of this disease in the pre-antibiotic era. ${ }^{3,4}$

Subsequent work of Maxwell Finland, Robert Austrian, and many others has provided a broad base of knowledge about pneumococci and the diagnosis, treatment, and prevention of pneumococcal infections based on then current scientific theories and advances. Even though pneumonia remains a frequent clinical diagnosis with significant morbidity and mortality, especially in children and older adults, many clinicians are unaware of advances in microbiology and clinical management and their relevance to 
public health. ${ }^{5}$ Specifically, the introduction of antibiotics and the development of vaccines against pneumococci were important milestones in modern infectious disease control. The emergence of antibiotic resistant pneumococci emphasizes the importance of genetic studies in bacteria, antibiotic development and management, epidemiology, including global studies, and vaccination programs. ${ }^{6}$ We have summarized the development of information about pneumococcal disease through the twenty editions of Harrison's Principles of Internal Medicine by comparing information in the $1^{\text {st }}$ edition published in 1950 with information in the $20^{\text {th }}$ edition published in 2018 .

Paul Beeson wrote the chapter on pneumococcal infections in the $1^{\text {st }}$ edition of Harrison's Principles of Internal Medicine published in $1950 .^{7}$ This chapter covered 7 pages of material related to pneumococcal pneumonia. Major sections included pneumococcal infections, pneumococcal pneumonia, pneumococcal meningitis, pneumococcal endocarditis, and pneumococcal peritonitis. David Goldblatt and Kathryn L. O'Brien wrote the chapter on pneumococcal infections in the $20^{\text {th }}$ edition of Principles of Internal Medicine published in $2018 .^{8}$ This chapter was approximately twice as long as the chapter in the $1^{\text {st }}$ edition; major sections included microbiology, epidemiology, pathogenesis, host defense mechanisms, clinical manifestations, treatment, prevention, and global health. There was much more information on bacterial virulence factors, host defense mechanisms, treatment, prevention, and global health in this edition. The chapter published in 1950 provides a description of a clinical disorder; the chapter published in 2018 provides a description of a disease process. ${ }^{9,10}$ The $20^{\text {th }}$ edition of Harrison's textbook also includes a long chapter on pneumonia and a chapter on disorders of the pleura. ${ }^{11,12}$

\section{MICROBIOLOGY, EPIDEMIOLOGY, AND PATHOGENESIS}

Beeson provided limited information in his chapter on the pneumococcus bacterium, the epidemiology of pneumococcal colonization and infection, and the pathogenesis of infection. ${ }^{7} \mathrm{He}$ stated that the mechanism for tissue damage by these bacteria is obscure.
Goldblatt and O'Brien included significant amounts of information on the bacterium, its virulence factors, and its epidemiology. ${ }^{8}$ They note that the uncertainty of any diagnosis of pneumonia in the absence of bacteremia increases the difficulty in establishing an exact microbiological diagnosis and that this, in turn, limits conclusions from epidemiology studies, clinical series, and vaccination programs. In addition, they noted that the development of varying levels of antibiotic resistance in different patient populations compromises the interpretation of outcomes, such as case fatality rates. Their discussion of host defense mechanisms included a paragraph on innate immunity and on acquired immunity.

\section{Clinical DESCRIPTION OF PNEUMOCOCCAL PNEUMONIA}

Beeson described pneumococcal pneumonia as a disease with remarkable uniformity. Patients present with the abrupt onset of a shaking chill, a rapid rise in temperature, severe pleuritic chest pain, and rusty-colored mucoid sputum. ${ }^{7}$ Examination of the patient's chest reveals splinting, increased tactile fremitus, and bronchial breath sounds. The patient often lies on the affected side to minimize respiratory motion; percussion on the affected side is dull with an increase in tactile fremitus. The patient's breathing is also shallow and painful, which may lead to cyanosis. In some cases, the patient is jaundiced and has herpetic lesions around the mouth. In some patients, death from pneumococcal pneumonia is associated with empyema and pericarditis.

Golblatt and O'Brien classified pneumococcal infections into two groups: noninvasive (e.g., otitis media) or invasive (e.g., bacteremic pneumonia, meningitis) according to whether a sterile site is infected. ${ }^{8}$ In either case, all pneumococcal infections result from colonization of the nasopharyngeal passages. The clinical description of pneumococcal pneumonia is similar to the $1^{\text {st }}$ edition; however, older patients with pneumococcal pneumonia are described as having less specific clinical presentations and needing prompt treatment to avoid increased morbidity and risk of death. Specifically, mental status changes, such as confusion, are common in older patients. This 
discussion makes use of the current understanding of the pathogenesis of respiratory infections and uses the terms of invasive and noninvasive.

\section{Complications of PNEUMOCOCCAL PNEUMONIA}

Pneumococcal pneumonia can cause serious extrapulmonary complications often but not always when antibiotic therapy has been delayed. In the $1^{\text {st }}$ edition, complications associated with pneumococcal pneumonia included atelectasis, pleural effusion, delayed resolution, brain abscess, pericarditis, arthritis, meningitis, and endocarditis. ${ }^{7}$ Patients with atelectasis typically presented with sudden onset pleuritic pain with rapid breathing and signs of distress; in older patients, atelectasis may persist for longer periods than in younger patients. Pleural effusions occur in approximately $5 \%$ of patients, but the fluid accumulation usually does not displace the heart and lungs significantly. Pneumococcal infections can lead to empyema in $5 \%-8 \%$ of patients. Unlike pleural effusions, the fluid accumulation from empyema can compress the lung leading to pleural scarring and limited chest movement. In severe cases, patients may have spontaneous drainage of the empyema through the chest wall.

Even with advances in the medical management of pneumococcal pneumonia, the $20^{\text {th }}$ edition reported that empyema was still a potential complication in $5 \%$ of patients. ${ }^{8}$ In this edition, empyema was described as being accompanied by fever and leukocytosis after 4-5 days of antibiotic treatment. If the pleural fluid shows frank pus, bacteria on Gram stain or culture, or a $\mathrm{pH}$ below 7.1 , drainage should be initiated using a chest tube. This section of the chapter provides a more detail about the characterization of parapneumonic pleural effusions than in the $1^{\text {st }}$ edition. Finally, pneumococcal infection can cause meningitis, sinusitis, and otitis media with the latter being more common in children.

\section{Prognostic factors in PNEUmococcal PNEUMONIA}

Prognostic factors associated with an increased mortality for pneumococcal pneumonia help clinicians identify patients at risk for poor outcomes. In the $1^{\text {st }}$ edition, Beeson described old age, circulatory failure, leukopenia, bacteremia, and pre-existing disease as poor prognostic factors in pneumococcal pneumonia. ${ }^{7}$ Without antibiotic treatment, the mortality from pneumococcal pneumonia was $25 \%-30 \%$; with penicillin therapy, the mortality rate dropped to $5 \%$. Mortality from pneumococcal pneumonia usually occurs in patients with underlying conditions, such as cancer, alcoholism, malnutrition, or other medical complications. The $20^{\text {th }}$ edition described leukopenia as a poor prognostic sign associated with fatal outcomes. ${ }^{8}$ In addition, HIV infection, the lack of breast feeding in infants and children, smoking, malnutrition, sickle cell disease, and limited access to medical care increase mortality.

The clinical presentation of pneumococcal pneumonia and its associated complications probably have not fundamentally changed over the last 70 years. However, increased longevity and associated comorbidity do affect the initial presentation, the disease course, the frequency of complications, and outcomes. Understanding these interactions requires large studies with complete lists of all relevant disease and clinical factors for analysis. In addition, the use of antibiotics almost certainly changes in the clinical course and outcomes.

\section{DiffERENTIAL DIAGNOSIS}

In the $1^{\text {st }}$ edition of Harrison's Principles of Internal Medicine, Beeson discussed the presentation of primary atypical pneumonia. His differential diagnosis also included Friedlander's bacillus pneumonia, staphylococcal pneumonia, hemolytic streptococcal pneumonia, tularemia, psittacosis, $Q$ fever, tuberculosis, and pulmonary infarction in the differential diagnosis and described the clinical differences between these diseases and pneumococcal pneumonia. ${ }^{7}$ In the $20^{\text {th }}$ edition, the differential diagnosis of pneumococcal pneumonia included other clinical disorders with acute presentations, including cardiac conditions such as myocardial infarction and heart failure, and abdominal disorders with pain such as cholecystitis, appendicitis, perforated peptic ulcer disease, and subphrenic abscesses. ${ }^{8}$ In addition, the differential diagnosis for pneumococcal pneumonia also includes pneumonia 
caused by viral pathogens, mycoplasmas, Haemophilus influenzae, Klebsiella pneumoniae, Staphylococcus aureus, Legionella pneumophila, and Pneumocystis jirovecii. ${ }^{8}$ This broader differential diagnosis reflects advances in the identification of viral pathogens and newly discovered bacterial pathogens.

\section{Diagnosis of PNEUMOCOCCAL PNEUMONIA}

In the early editions of Harrison's textbook, the importance of sputum Gram stains in the diagnosis of pneumococcal pneumonia was repeatedly stressed. In the $1^{\text {st }}$ edition, Beeson stated that when sputum Gram stains should reveal polymorphonuclear leukocytes and moderate numbers of Gram positive cocci in pairs. ${ }^{7}$ The authors of the $20^{\text {th }}$ edition summarize information on the Gram stain in two sentences and note that its usefulness depends on the quality of the sputum specimen and prior antibiotic therapy. ${ }^{8}$ Routine laboratory methods using sputum Gram stains and cultures frequently do not establish the etiology of a bacterial pneumonia. In addition, conventional methods for diagnosing pneumonia require time for the identification of the bacteria and then additional time for antibiotic sensitivity studies. These methods may not identify co-infections.

Patients with pneumococcal pneumonia often have a homogenous density in the infected lung or lobes on chest $\mathrm{x}$-ray. The $20^{\text {th }}$ edition of Harrison's textbook stated that the gold standard for etiologic diagnosis of pneumococcal pneumonia was direct examination of the lung tissues (which is almost never done in routine clinical practice). ${ }^{8}$ In most cases, an x-ray of the lung shows lobar, segmental, or patchy consolidation; in $30 \%$ of cases, more than one lobe of the affected lung is involved. For children, an x-ray shows a "round pneumonia," a distinct spherical consolidation that is uncommon in adults. However, pneumococcal pneumonia can occur without infiltrates on $\mathrm{x}$-ray in the early course of the illness or with dehydration.

Blood cultures growing pneumococci usually establish this diagnosis. Supportive but nonspecific information includes elevated polymorphonuclear leukocyte counts, leukopenia, and elevated liver enzyme tests, such hyperbilirubinemia. ${ }^{8}$ Furthermore, anemia, low serum albumin levels, hyponatremia, and elevated serum creatinine levels occur in $2 \%-30 \%$ of pneumococcal pneumonia patients.

Newer tests include urinary pneumococcal antigen assays that support the diagnosis of pneumococcal pneumonia; however, the results can be confounded by pneumococcal colonization in the nasopharyngeal passages. ${ }^{8}$ In most cases, a positive pneumococcal urinary antigen test has a high predictive value for pneumococcal pneumonia due to the low prevalence of $S$. pneumoniae in the nasal passages. In low income communities with more crowding, more frequent colonization with $S$. pneumoniae in the upper airways makes urinary antigen tests less useful. A recent advance to overcome these limitations has been the development of quantitative serotype-specific urinary antigen detection assays that can identify pneumococcal serotypes which usually cause clinical disease and rarely occur as asymptomatic colonizers. ${ }^{8}$

Clinical laboratories can now provide more rapid identification of Streptococcus pneumoniae based on nucleic acid analysis using real-time polymerase chain reaction tests. ${ }^{13}$ These tests can be used with blood samples and respiratory specimens and can also rapidly identify genes associated with penicillin resistance. ${ }^{14}$ These tests have the potential to provide a more secure diagnosis and better antibiotic selection early in the disease course. This technology was not discussed in the $20^{\text {th }}$ edition.

\section{TREATMENT OF PNEUMOCOCCAL INFECTIONS}

In the modern era, treatment of pneumococcal pneumonia has become more complicated and potentially difficult given the increase in antibiotic resistant pneumococci. Before antibiotics, pneumococcal pneumonia patients were encouraged to lie in the supine position and were allowed to turn and sit for examination and procedures. Oxygen therapy was initiated if the patient showed intense cyanosis. ${ }^{7}$ In the $1^{\text {st }}$ edition, Beeson wrote that "penicillin is the drug of choice in the treatment of pneumococcal pneumonia." ${ }^{\prime 7}$ Specifically, penicillin therapy should begin with 60,000 units (1 $\mathrm{mg}$ of penicillin sodium equals 1667 units of penicillin) at three to four hour intervals with 
a total dose of 300,000 units per day. Treatment with penicillin should be continued for two to three days after the patient is afebrile. Serum therapy was also described as an effective treatment but was subsequently abandoned due to its expense, its difficulty to administer, and the risk of anaphylaxis and serum sickness. Aureomycin was also effective in some patients but was considered second line therapy with a recommended starting dose between 1.5 to 6 grams per day. If penicillin was ineffective, sulfonamides were also recommended as a second line therapy; the starting dose is 6 grams per day. For empyemas associated with pneumococcal pneumonia, Beeson recommended that 200,000 units of penicillin should be injected into the exudate after partial removal of the empyema; this process should be repeated daily until cultures were negative. Other recommendations included bedrest, codeine, adhesive strapping, and intercostal nerve block for pleuritic pain.

In the $20^{\text {th }}$ edition, penicillin $\mathrm{G}$ was described as the classic choice of antibiotics for pneumococcal pneumonias with daily doses ranging from $50,000 \mathrm{U} / \mathrm{kg}$ for minor infections to $300,000 \mathrm{U} / \mathrm{kg}$ for meningitis. ${ }^{8}$ Although $\beta$-lactam drugs, such as ampicillin, cefotaxime, ceftriaxone, and cefuroxime, can be used to treat pneumococcal pneumonia, these drugs provide little advantage over penicillin. Given the increase in penicillin resistant pneumococci, the treatment decisions in patients with pneumococcal pneumonia require careful review of all relevant factors. Pneumococci resistant to macrolides and tetracyclines were first reported in the mid-1960s. Clindamycin, tetracycline, and trimethoprim-sulfamethoxazole are effective against pneumococci, but resistance can be encountered in different regions of the world. In most cases, penicillin resistance results from alterations in penicillin-binding protein (PBP) genes through transformation or horizontal transfer of genes between streptococcal species. As a result, the guidelines for antibiotic therapy for pneumococcal pneumonia differ depending on local resistance patterns to antibiotics.

For adults, amoxicillin is the first-line treatment for outpatient pneumococcal pneumonia management, which should be given for a minimum of five days. ${ }^{8}$ Antibiotic alternatives, such as levofloxacin, moxifloxacin, clindamycin, azithromycin, or clarithromycin, can also be given. However, higher levels of resistance have been reported with azithromycin.

Advances in medical care allowed the authors of the $20^{\text {th }}$ edition to provide much more information about treatment than Beeson did. They report the frequency of resistance to penicillin and the genetic basis for this resistance and describe standardized laboratory tests to determine the minimal inhibitory concentration for antibiotics for more uniform reporting. They also note that several guidelines provide up-to-date information about treatment decisions.

\section{THE PNEUMOCOCCAL VACCINE}

A pneumococcal vaccine was not available at the time of the $1^{\text {st }}$ edition and was introduced into the United States in 1983. The $20^{\text {th }}$ edition emphasized the poor response of infants and children to capsular polysaccharide vaccines (PPSV23) and discussed new clinical trials directed at this target population. ${ }^{8}$ Furthermore, it remains controversial whether the PPSV23 vaccine decreases all-cause mortality for pneumococcal pneumonia given the conflicting conclusions in several meta-analyses on its efficacy. It is generally agreed that the PPSV23 vaccine is extremely effective in the prevention of invasive pneumococcal disease but is less effective or ineffective against nonbacteremic pneumococcal pneumonia. Furthermore, PPSV23 is less effective in older or immunodeficient patients who have reduced antibody responses. Two new polysaccharide-protein conjugate vaccines against 10 serotypes and 13 serotypes (PCV10 and PCV13) have been developed. Compared to the PPSV23 vaccines, the PCV 10 and 13 vaccines protect children against invasive pneumococcal disease and antibiotic resistant pneumococcal species. ${ }^{8}$ Clinical trials with PCV10 and 13 were effective against pneumonia, otitis media, and nasopharyngeal colonization and reduced all-cause mortality.

The authors of the $20^{\text {th }}$ edition note that the serotypes used in vaccines are more frequently resistant to antibiotics than the non-vaccine serotypes and that vaccination has reduced the frequency of infections 
with antibiotic resistant pneumococci. They also noted that up-to-date recommendations for vaccination come from the Advisory Committee on Immunization Practices from the CDC. Other measures which might reduce the frequency or severity of pneumococcal disease include vaccination against influenza and antibiotic stewardship which can reduce the frequency of antibiotic resistance.

\section{Discussion}

Comparison of the chapter on pneumococcal infections in $1^{\text {st }}$ edition of Harrison's Principles of Internal Medicine with the chapter in the $20^{\text {th }}$ edition easily demonstrates the significant increase in information about this bacterium, its pathogenesis, its treatment, and its prevention. Several questions highlight these changes and allow predictions regarding future challenges with these infections.

Has the bacterium changed over the last 70 years? Clearly the introduction of antibiotics has made a substantial difference in treatment options but has also increased selection pressure on bacterial populations to develop antibiotic resistance. Recent studies indicate that a significant percentage of pneumococci are resistant to penicillin and macrolides. For example, Hawkins et al. measured antibody resistance determinants and susceptibility in pneumococcal isolates recovered in Trinidad and Tobago. ${ }^{15}$ They found that $42 \%$ of the isolates were predicted to be resistant to at least one antimicrobial class and $13 \%$ were predicted to be resistant to at least 3 classes. This is likely explained by the widespread use of antibiotics and suggests that antibiotic stewardship is essential to slow this process. However, it is unclear whether or not antibiotic resistance has important effects on outcomes even when the patient receives an antibiotic to which the isolate is resistant. ${ }^{16-18}$ The development of vaccines has changed the prevalence of certain serotypes identified in infected patients and possibly the prevalence of antibiotic resistance. There are fewer infections by vaccine serotypes, but replacement serotypes have increased in frequency and now cause infections. The pneumococcal capsule is the basis of serotyping and is a key factor in pneumococcal virulence, and there are studies that demonstrate that bacteria can have capsule switching through gene transformation which could influence virulence. ${ }^{19}$ This indicates there are dynamic changes in serotype distribution independent of the effects of vaccination.

Are there important changes in pathogenesis? Pneumococcal infections develop following nasal colonization, and multiple factors unrelated to bacterial virulence can affect colonization. For example, colonization increases following viral infection such as influenza and can increase secondary to pollution..$^{20-22}$ Consequently, the rates of infection can change during periods of increased viral activity and during periods of increased pollution independent of changes in serotype distribution, bacterial virulence, or antibiotic resistance.

Has the clinical presentation of pneumococcal pneumonia changed? Aging patients typically have more comorbidities, and these influence the clinical presentation during acute infections and increase the complication rate and case fatality rate. In particular, diseases, such as congestive heart failure, chronic obstructive pulmonary disease, diabetes, and endstage renal disease, affect the frequency and severity of pneumococcal infections. Cilloniz et al. prospectively studied pulmonary complications, including pleural effusion, empyema, and multilobar pneumonia, in adults with pneumococcal pneumonia admitted to a hospital in Spain. ${ }^{23}$ Thirty-eight percent of these patients had complications and had higher rates of intensive care unit admission, higher rates of shock, and longer lengths of stay. However, they had mortality rates similar to patients without complications, and they had lower rates of penicillin resistance. Chronic liver disease, high C-reactive protein levels, and high creatinine levels predicted complications. The diagnosis of COPD did not predict these complications. Therefore, the complication rate with these infections depends on the characteristics of infected patients in addition to bacterial virulence.

What information is needed to provide an overview of the frequency and severity of pneumococcal infections in various populations? This problem starts with difficulty in identifying the etiology of pneumonias without bacteremia. Pneumonia frequently occurs in patients who do not have positive sputum cultures. In addition, if Streptococcus pneumoniae is isolated 
on culture, it may not be further characterized using serotyping. Consequently, changes in the bacterial populations over time are not tracked. The CDC has developed a multiplex system which can identify serotypes and genes for antibiotic resistance. ${ }^{13}$ Periodic surveys of isolates provide information necessary to understand changes in bacterial populations. Analysis of the interaction between comorbidity and pneumococcal infection depends on a secure bacterial diagnosis and on a complete list of comorbidities and their severity. This information, especially the severity of a particular comorbidity, is relatively difficult to obtain. For example, hospital discharge data do not provide information about the severity of COPD but only document its presence as a discharge diagnosis. This complicates any analysis trying to determine whether outcomes reflect differences in bacterial virulence or in comorbidity.

Finally, does global health matter? The antibiotic sensitivity and the serotypes of pneumococci isolated in other parts of the world may differ significantly from pneumococci isolated in United States. Given the ease of international travel, bacteria in one region of the world can easily move into other regions. This creates situations in which population based studies in the United States may not be relevant to ongoing infections in either travelers or contacts of these travelers.

\section{Conclusion}

Since the $1^{\text {st }}$ edition of Harrison's textbook in 1950, information about epidemiology, pathogenesis, clinical presentation, diagnosis, treatment, and prevention of pneumococcal infection has continuously increased with scientific, technological, and medical advances. The development of antibiotics and vaccines has reduced morbidity and mortality in children and older patients. However, widespread antibiotic use has produced antibiotic-resistant pneumococci, which continue to challenge current treatment of pneumococcal pneumonia. As documented in the Harrison's Principles of Internal Medicine, the historical developments in pneumococcal pneumonia provide important insights into infectious disease and help predict new challenges that will likely appear in the future. These challenges will include the need for new antibiotics, avoidance of antibiotic overuse, comprehensive immunization programs, and accurate identification of bacterial pathogens using technology based on polymerase chain reaction testing. This review also demonstrates the value of information in textbooks that have a long publication history and multiple editions with chapters written by experts.

Article citation: Kopel JJ, Hakim A, Nugent K, Berk SL. Pneumococcal pneumonia-a history based on chapters from the first edition (1950) and the latest edition (2018) of Harrison's Principles of Internal Medicine. The Southwest Respiratory and Critical Care Chronicles 2021;9(37):24-31

From: Department of Internal Medicine, Texas Tech University Health Sciences Center, Lubbock, Texas

Submitted: $12 / 10 / 2020$

Accepted: $1 / 6 / 2021$

Reviewer: Jacob Nichols MD

Conflicts of interest: none

This work is licensed under a Creative Commons Attribution-ShareAlike 4.0 International License.

\section{REFERENCES}

1. Pasteur L. Sur une maladie nouvelle provoquée par la salive d'un enfant mort de rage. Comptes Rendus de l'Académie des Sciences de Paris 1881;92:159.

2. Sternberg GM. A fatal form of septicaemia in the rabbit produced by the subcutaneous injection of human saliva. An experimental research. Joseph Meredith Toner Collection (Library of Congress); National Board of Health (U.S.). John Murphy and company, Baltimore, April 30, 1881.

3. White B. The Biology of the Pneumococcus. Cambrdige, Massachusetts: The Commonwealth Fund, 1938.

4. Heffron R. Pneumonia with Special Reference to Pneumococcus Lobar Pneumonia. Cambridge, Massachusetts: Harvard University Press, 1939.

5. Scally G. The importance of the past in public health. J Epi Comm Health 2004;58:751-755.

6. Podolsky SH. The changing fate of pneumonia as a public health concern in 20th-century america and beyond. Am J Public Health 2005;95:2144-54.

7. Beeson P. Pneumococcal Infections. In: Harrison T, Beeson P, Resnick W, Thorn G, Wintrobe M, editorss. Harrison's Principles Internal Medicine. First ed. Philadelphia, Pennsylvania: Blakiiston Company; 1950:789-95. 
8. Goldblatt D, O'Brien K. Pneumococcal Infections. In: Longo D, Fauci A, Kasper D, Hauser S, Jameson J, Loscalzo $\mathrm{J}$, editors. Harrison's Principles of Internal Medicine. New York, New York: McGraw-Hill Co., 2018: Chapter 141.

9. Whitbeck C. Causation in medicine: the disease entity model. Philosophy of Science 1977:619-37.

10. Lesson 1: Introduction to Epidemiology. Section 9: Natural History and Spectrum of Disease. https://www.cdc.gov/csels/ dsepd/ss1978/lesson1/section9.html. Accessed 5/3/2020

11. Mandell LA, Wunderink R. Pneumonia. In: Longo D, Fauci A, Kasper D, Hauser S, Jameson J, Loscalzo J, editors. Harrison's Principles of Internal Medicine. New York, New York: McGraw-Hill Co., 2018: Chapter 121.

12. Light RW. Pleural effusion. In: Longo D, Fauci A, Kasper D, Hauser S, Jameson J, Loscalzo J, editors. Harrison's Principles of Internal Medicine. New York, New York: McGrawHill Co., 2018: Chapter 288.

13. Velusamy S, Tran T, Mongkolrattanothai $\mathrm{T}$, et al. Expanded sequential quadriplex real-time polymerase chain reaction (PCR) for identifying pneumococcal serotypes, penicillin susceptibility, and resistance markers. Diagnostic Micro Infect Dis 2020:115037.

14. Zettler EW, Scheibe RM, Dias CAG, et al. Determination of penicillin resistance in Streptococcus pneumoniae isolates from southern Brazil by PCR. International J Infect Dis 2006;10:110-5.

15. Hawkins PA, Akpaka PE, Nurse-Lucas M, et al. Antimicrobial resistance determinants and susceptibility profiles of pneumococcal isolates recovered in Trinidad and Tobago. J Global Antimicrobial Resistance 2017;11:148-51.

16. Yin Y, Wunderink R. Antibiotic resistance in communityacquired pneumonia pathogens. Seminars Resp Crit Care Medicine 2016;37:829-38.

17. Cilloniz C, Albert RK, Liapikou A, et al. The effect of macrolide resistance on the presentation and outcome of patients hospitalized for Streptococcus pneumoniae pneumonia. Am J Resp Crit Care Med 2015;191:1265-72.

18. Cheng AC, Jenney AWJ. Macrolide resistance in pneumococci-is it relevant? Pneumonia 2016;8.

19. Wyres KL, Lambertsen LM, Croucher NJ, et al. Pneumococcal capsular switching: a historical perspective. J Infect Dis 2012;207:439-49.

20. Chao Y, Marks LR, Pettigrew MM, et al. Streptococcus pneumoniae biofilm formation and dispersion during colonization and disease. Frontiers Cell Infect Micro 2015;4.

21. Pedro-Botet ML, Burgos J, Luján M, et al. Impact of the 2009 influenza A H1N1 pandemic on invasive pneumococcal disease in adults. Scand J Infect Dis 2014;46:185-92.

22. Hussey SJK, Purves J, Allcock N, et al. Air pollution alters Staphylococcus aureus and Streptococcus pneumoniae biofilms, antibiotic tolerance and colonisation. Environ Micro 2017;19:1868-80.

23. Cillóniz C, Ewig S, Polverino E, et al. Pulmonary complications of pneumococcal community-acquired pneumonia: incidence, predictors, and outcomes. Clin Micro Infect 2012; 18:1134-42. 